\title{
Anomalous isotopic effect near the charge-ordering quantum criticality
}

\author{
S. Andergassen, S. Caprara, C. Di Castro, and M. Grilli \\ Istituto Nazionale per la Fisica della Materia - Unità di Roma 1, and Dipartimento di Fisica - Università di Roma "La \\ Sapienza", Piazzale Aldo Moro 2, I-00185 Roma, Italy
}

\begin{abstract}
Within the Hubbard-Holstein model, we evaluate the various crossover lines marking the opening of pseudogaps in the cuprates, which, in our scenario, are ruled by the proximity to a charge-ordering quantum criticality (stripe formation). We provide also an analysis of their isotopic dependencies, as produced by critical fluctuations. We find no isotopic shift of the temperature $T^{0}$ marked as a reduction of the quasiparticle density of states in various experiments, and a substantial positive shift of the pseudogap-formation temperature $T^{*}$. We infer that the superconducting critical temperature $T_{c}$ has almost no shift in the optimally- and overdoped regimes while it has a small negative isotopic shift in the underdoped, which increses upon underdoping. We account also for the possible dynamical nature of the charge-ordering transition, and explain in this way the spread of the values of $T^{*}$ and of its isotopic shift, obtained with experimental probes having different characteristic timescales.
\end{abstract}

PACS: 71.10. w, 71.28.+d, 74.72. h, 71.45.Lr

There are several experimental evidences [1] that the peculiar properties of the cuprates, both in the normal and in the superconducting phase are controlled by a Quantum Critical Point (QCP), located near the optimal doping $\delta=\delta_{o p t}$. In this framework the phase diagram of the cuprates is naturally partitioned into a (nearly) ordered, a quantum critical, and a quantum disordered region corresponding to the under-, optimally, and overdoped regions respectively. The ordered region occurs below a second-order transition line $T_{\text {? }}(\delta)$, which depends on the nature of the underlying ordering and, upon increasing $\delta$, ends at $T=0$ in a $\mathrm{QCP}\left(\right.$ at $\delta=\delta_{c} \gtrsim \delta_{\text {opt }}$ ). The above correspondence between the theoretical and experimental partitioning of the phase diagram, leads to a close connection [1] between the hypothetical $T_{?}(\delta)$ and the crossover line $T^{*}(\delta)$, below which a pseudogap behavior is observed in NQR, NMR relaxation-rate, XANES, and ARPES measurements [see, e.g., Ref. [2] for a recent overview on $\mathrm{La}_{2-\delta} \mathrm{Sr}_{\delta} \mathrm{CuO}_{4}$ (LSCO)]. In the proximity of quantum criticality, critical fluctuations mediate a singular interaction among the quasiparticles which can account for both the violation of the Fermi-liquid behavior observed in the normal phase of the cuprates, and the strong pairing mechanism leading to high- $T_{c}$ superconductivity [1] 3 5]. Various realizations of this scenario have been proposed, associated with different quantum criticalities, e.g., antiferromagnetic [3], excitonic [ [4, change in the symmetry of the superconducting order parameter [6], or incommensurate charge-density wave [5].

In the QCP framework, any mechanism shifting the position of the QCP is mirrored by corresponding shifts in $T_{?}(\delta)$ and in the superconducting critical line $T_{c}(\delta)$. In particular, the observation of isotopic effects (IE's) on $T_{c}$ [7, 8] and on $T^{*}$ 9 11] suggests that a lattice mechanism underlies the instability marked by the QCP. We here consider the single-band Hubbard-Holstein model as a minimal model to describe the strongly correlated electrons coupled to the lattice, giving rise to a QCP for the onset of a phonon-induced incommensurate charge ordering (CO) [5, 12, 13]. This introduces density inhomogeneities on a semimicroscopic scale, and corresponds to the onset of stripes 14], coming from the high-doping regime. Thus, in our approach, the critical line $T_{?}(\delta)$ corresponds to the line for $\mathrm{CO}, T_{C O}(\delta)$, which in real materials can be masked by pair formation and lattice effects.

In this letter, we first determine the mean-field (m-f) critical line $T_{C O}^{(0)}(\delta)$, which we identify with the weakpseudogap crossover line $T^{0}(\delta)\left[\gg T^{*}(\delta)\right]$ observed in Knight-shift, transport, and static susceptibility measurements [2], as the incipient depression of the singleparticle density of states (DOS). Indeed, for $T<T_{C O}^{(0)}$ one expects the $\mathrm{CO}$ fluctuations to become substantial, leading to a reduction of the quasiparticle DOS, which accounts for the weak-pseudogap behavior. Then, we investigate the effect of fluctuations near the CO QCP in order to determine: a) the fluctuation-corrected critical line $T_{C O}(\delta)$, which we relate to the pseudogap crossover line $T^{*}(\delta)$; b) the IE on $T_{C O}(\delta)$, from which we shall also infer the effect on $T_{c}(\delta)$. In particular we shall describe the highly non-trivial effect of quantum criticality in determining IE's on $T^{*}$ and $T_{c}$, which can be strong and weak, respectively, and opposite in sign in the underdoped cuprates. We shall also show why both $T^{*}$ and its isotopic shift are observed to be larger in experiments with shorter characteristic timescales. The near absence of IE on $T_{c}$ near and above $\delta_{\text {opt }}$ will also be naturally accounted for.

- The model and the mean-field instability - Our two-dimensional (2D) Hamiltonian is

$$
\begin{aligned}
H & =-t \sum_{\langle i, j\rangle, \sigma}\left(c_{i \sigma}^{\dagger} c_{j \sigma}+H . c .\right)-t^{\prime} \sum_{\langle\langle i, j\rangle\rangle, \sigma}\left(c_{i \sigma}^{\dagger} c_{j \sigma}+H . c .\right) \\
& -\mu_{0} \sum_{i \sigma} n_{i \sigma}+U \sum_{i} n_{i \uparrow} n_{i \downarrow}+\frac{1}{2} \sum_{\mathbf{q} \neq 0} V_{C}(\mathbf{q}) \rho_{\mathbf{q}} \rho_{-\mathbf{q}}
\end{aligned}
$$




$$
+\omega_{0} \sum_{i} a_{i}^{\dagger} a_{i}+g \sum_{i, \sigma}\left(a_{i}^{\dagger}+a_{i}\right)\left(n_{i \sigma}-\left\langle n_{i \sigma}\right\rangle\right),
$$

where $c_{i \sigma}^{(\dagger)}$ are the fermion operators, $a_{i}^{(\dagger)}$ are the phonon operators, $\langle i, j\rangle$ and $\langle\langle i, j\rangle\rangle$ indicate nearest- and nextnearest-neighbor sites, coupled by the hopping parameters $t$ and $t^{\prime}$ respectively. The lattice spacing has been set to unity. The chemical potential $\mu_{0}$ is coupled to the local electron density $n_{i \sigma} \equiv c_{i \sigma}^{\dagger} c_{i \sigma}, U$ is the on-site Hubbard repulsion, and $\rho_{\mathbf{q}} \equiv \sum_{\mathbf{k}, \sigma} c_{\mathbf{k}+\mathbf{q}, \sigma}^{\dagger} c_{\mathbf{k}, \sigma} . V_{C}(\mathbf{q})\left(\approx V_{C} /|\mathbf{q}|\right.$ at small $|\mathbf{q}|)$ is the Coulomb interaction between electrons in a 2D plane embedded in the three-dimensional (3D) space [5, 13]. $\omega_{0}$ is the phonon frequency and $g$ is the Holstein electron-phonon coupling. In the limit $U \rightarrow \infty$, this model was solved with a standard slave-boson technique, at leading order within a large- $\mathrm{N}$ expansion in Refs. [5, 13]. The most relevant result was that a $\mathrm{CO}$ instability with a finite wavevector $\mathbf{q}_{c}$, incommensurate with the underlying lattice, was found at $T=0$ when $\delta$ is reduced below a critical value $\delta_{c}^{(0)}$. Besides a small, weakly momentum-dependent, residual repulsion, nearby this instability the critical charge fluctuations mediate a singular scattering

$$
\Gamma\left(\mathbf{q}, \omega_{n}\right) \approx-\frac{V}{\xi_{0}^{-2}+\left|\mathbf{q}-\mathbf{q}_{c}\right|^{2}+\gamma\left|\omega_{n}\right|},
$$

of strength $V$, between the quasiparticles. Here $\omega_{n}$ is a bosonic Matsubara frequency, $\gamma \sim t^{-1}$ is a characteristic timescale, and $\xi_{0}^{-2}$ is the $\mathrm{m}-\mathrm{f}$ inverse square correlation length, which measures the distance from criticality. Eq. (2) displays the behavior of a Gaussian QCP with a dynamical critical index $z=2$ [15].

Quite remarkably, the complicated formal structure of the quasiparticle scattering, mediated by slave bosons, phonons and by the Coulomb interaction, is well represented near criticality by a RPA resummation $\Gamma\left(\mathbf{q}, \omega_{n}\right) \approx$ $V_{e f f}(\mathbf{q}) /\left[1+V_{e f f}(\mathbf{q}) \Pi\left(\mathbf{q}, \omega_{n}\right)\right]$ of an effective static interaction $V_{\text {eff }}(\mathbf{q})=\tilde{U}(\mathbf{q})+V_{C}(\mathbf{q})-\lambda t$ [16]. Here $\Pi$ is the fermionic polarization bubble in a $2 \mathrm{D}$ lattice, $\lambda \equiv 2 g^{2} / t \omega_{0}$ is the dimensionless electron-phonon coupling, and $\tilde{U}(\mathbf{q}) \simeq A+B|\mathbf{q}|^{2}$ is the residual short-range repulsion between quasiparticles. For the correspondence of $V_{e f f}(\mathbf{q})$ with the parameters of Eq. (11) see Ref. [16]. The instability condition, which occurs for reasonable values $\lambda \sim 1$, is $1+V_{\text {eff }}\left(\mathbf{q}_{c}\right) \Pi\left(\mathbf{q}_{c}, \omega=0\right)=0$. At $T=0$ this determines $\mathbf{q}_{c}$ and the position of the m-f QCP $\delta_{c}^{(0)}$. For realistic parameters we find $\mathbf{q}_{c} \approx( \pm 1,0)$ or $(0, \pm 1)$, and $\delta_{c}^{(0)} \approx 0.2[5,13]$. By expanding $1+V_{\text {eff }} \Pi$ near the instability at $T=0$, we find $\xi_{0}^{-2} \propto \delta-\delta_{c}^{(0)}$, which gives the Gaussian index $\nu=1 / 2$ for $\xi_{0}$. The $\mathrm{m}-\mathrm{f}$ critical line $T_{C O}^{(0)}(\delta)$ (the solid-line curve in Fig. 1), which starts from the m-f QCP at $\delta_{c}^{(0)}$ is obtained by considering the $T$ dependence of the bare polarization bubble, which reduces to the simple Fermi-liquid form, $\propto T^{2}$, at low $T$. We identify this $\mathrm{m}-\mathrm{f}$ transition with the experimental crossover line $T^{0}$, extrapolating to low $T$ at a doping, which we identify with our $\delta_{c}^{(0)}$. From the data reported in Ref. [2], for LSCO we estimate $\delta_{c}^{(0)} \approx 0.22$. Then we evaluate $T_{C O}^{(0)}(\delta)$ by taking standard quasiparticle (i.e., dressed by the slave bosons) band parameters $\left(t_{q p}=0.2 \mathrm{eV}, t_{q p}^{\prime}=-0.05 \mathrm{eV}, \omega_{0}=0.07 \mathrm{eV}\right.$, leading to $A=0.2 \mathrm{eV}$ and $B=0.17 \mathrm{eV}$ in the residual repulsion $\tilde{U}) . V_{C}=0.22 \mathrm{eV}$ and $g=0.21 \mathrm{eV}$ are adjusted to match the experimental extrapolation of $T^{0}$ with the $T=0 \mathrm{~m}-\mathrm{f}$ instability (i.e. $\left.\delta_{c}^{(0)}\right)$. We point out that the agreement between $T_{C O}^{(0)}(\delta)$ and $T^{0}(\delta)$ at finite $T$ is obtained without any further adjustment of the parameters. Similar parameters are taken for $\mathrm{Bi}_{2} \mathrm{Sr}_{2} \mathrm{CaCu}_{2} \mathrm{O}_{8}$ (Bi2212) to fit the data in Refs. 17, 18], with $V_{C}=0.22 \mathrm{eV}$ and $g=0.23$ $\mathrm{eV}$.
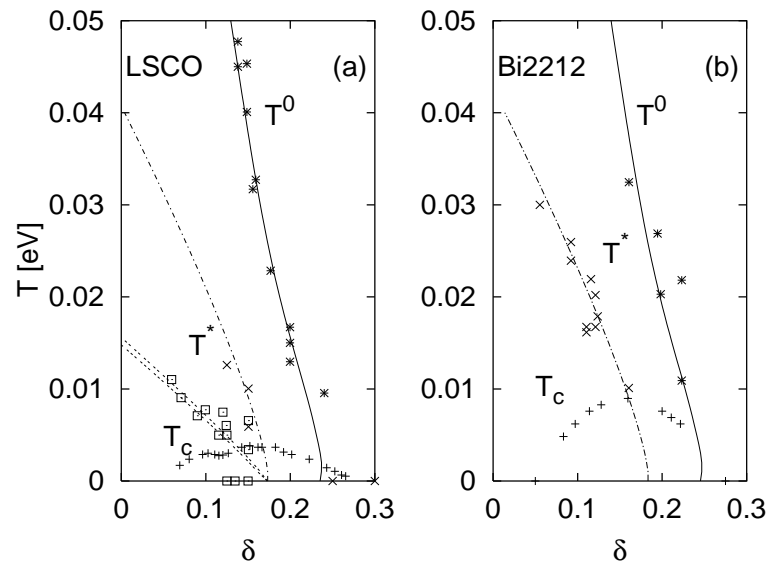

FIG. 1. The phase diagram of the cuprates according to the CO-QCP scenario for LSCO (a) and Bi2212 (b). The solid line is the $\mathrm{m}$-f critical line ending at $T=0$ in the $\mathrm{m}-\mathrm{f} \mathrm{QCP}$ at $\delta_{c}^{(0)}$. The lowest dashed line in panel (a) marks the 3D critical line in the presence of fluctuations, ending in the QCP at $\delta_{c}$. We took $\Omega_{\perp}=1 \mathrm{meV}$ (see text). The dot-dashed line in panels $(\mathrm{a}, \mathrm{b})$ indicates the "dynamical instability" condition (see text) for $\omega_{\text {probe }}=1 \mathrm{meV}$. The intermediate dashed line in panel (a) represents the "dynamical instability" condition for $\omega_{\text {probe }}=1 \mu \mathrm{eV}$. The experimental points for $T^{0}(*)$ and for $T^{*}$ measured with fast $(\times)$ and slow $(\square)$ probes for LSCO are from Ref. [2], those for Bi2212 are from Refs. 17, 18]. The experimental critical temperatures $T_{c}$ are also shown (+).

- The phase diagram beyond mean-field - The fluctuations shift the m-f QCP and critical line to their actual position 19.20 . Specifically the fluctuations which mediate the effective critical interaction, Eq. (2), can be included in the polarization bubble via the diagrams of Fig. 2a, leading to corrections beyond RPA. From the explicit evaluation of these diagrams we find the (selfconsistent) correction to the mass term $m \equiv \gamma^{-1} \xi^{-2}$ of the fluctuation propagator, Eq. (2), 


$$
m=m_{0}+12 u T \sum_{\left|\omega_{n}\right|<\omega_{0}} \sum_{\mathbf{q}} D\left(\mathbf{q}, \omega_{n} ; m\right),
$$

where $D=\left(\Omega_{\mathbf{q}}+\left|\omega_{n}\right|\right)^{-1}$ is the charge-fluctuation critical propagator, $u \sim V^{2} /\left(\gamma^{2} t^{3}\right)$ is the coupling resulting from the two 4-leg vertices represented in Fig. 2b. The critical modes have a 2D dispersion $\Omega_{\mathbf{q}}=\gamma^{-1}\left|\mathbf{q}-\mathbf{q}_{c}\right|^{2}+$ $m$, up to an ultraviolet bandwidth cutoff $\Omega_{\max } \sim \gamma^{-1}$, resulting from the underlying lattice. Since we deal with a phonon-driven $\mathrm{CO}$ instability, as it also follows from the detailed dynamical analysis of Ref. [13], $\omega_{0}$ appears as the ultraviolet frequency cut-off. By introducing the $\operatorname{DOS} N(\Omega)=\sum_{\mathbf{q}} \delta\left(\Omega-\Omega_{\mathbf{q}}\right)$ and the spectral-density representation of $D$, we rewrite Eq. (3) in the form

$$
\begin{aligned}
m & =m_{0}+\frac{24 u}{\pi} \int_{m}^{\Omega_{\max }} d \Omega N(\Omega) \int_{0}^{\infty} d z \frac{z}{z^{2}+\Omega^{2}} \\
& \times\left[b(z)+\frac{1}{2}-\frac{1}{\pi} \operatorname{arctg} \frac{z}{\omega_{0}}\right],
\end{aligned}
$$

where $b(z)=[\exp (z / T)-1]^{-1}$ is the Bose function. At $T=0$, Eq. (बi) with $m=0$ leads to a finite shift of the 2D QCP $\delta_{c}^{(0)}-\delta_{c} \propto \omega_{0}$ (see Fig. 1). At finite $T$, as a consequence of a constant DOS for the $2 \mathrm{D}$ modes, the integral in Eq. (4) is logarithmically divergent for $m \rightarrow 0$, leading to a vanishing of the renormalized $T_{C O}$ (MerminWagner theorem). This divergency is removed by considering the more realistic anisotropic 3D character of the critical fluctuations, introducing a small energy scale $\Omega_{\perp}$, below which the mode DOS is no longer constant, and displays a 3D square-root behavior $N\left(\Omega<\Omega_{\perp}\right) \sim \sqrt{\Omega}$ at criticality. This is enough to make the integral in Eq. (函 convergent and allows to determine the critical line (Eq. (位) with $m=0$ ) in the anisotropic 3D case as reported in Fig. 1a (lowest dashed line). The inclusion of fluctuations brings the critical line from temperatures of the order of typical electronic energies $\left(T_{C O}^{(0)} \sim t\right)$ down to much lower temperatures $T_{C O}$ of the order of the observed $T^{*}$ 's. Indeed, within the CO-QCP scenario, the pseudogap arises at $T<T^{*}$ because the quasiparticles feel an increasingly strong attractive interaction by approaching the critical line $T_{C O}(\delta)$. In the particle-hole channel, this interaction can produce a gap due to the incipient $\mathrm{CO}$. At the same time in the particle-particle channel, the strong attraction can lead to pair formation even in the absence of phase coherence. Therefore $T^{*}(\delta)$ closely tracks the underlying transition line $T_{C O}(\delta)$.

On the other hand, the spread in the measured values of $T^{*}$ depending on the experimental probe (see, e.g., Ref. [2]), indicates that the CO instability may be "dynamical", $\Omega_{\perp}$ being smaller than (or comparable to) typical frequencies of the experimental probes, $\omega_{\text {probe. }}$ In this case the self-consistency condition (4) includes $\omega_{\text {probe }}$ as the infrared cut-off in the integral over $\Omega, m$ being replaced by $\omega_{\text {probe }}$. This determines the doping and temperature dependence of the dynamical instability lines.
Two examples are reported in Fig. 1 for $\omega_{\text {probe }}=1 \mathrm{meV}$ (dot-dashed line if Fig. 1a,b), as in typical neutron scattering experiments, and $\omega_{\text {probe }}=1 \mu \mathrm{eV}$ (second dashed line from bottom in Fig. 1a), as in static experiments (NQR, NMR). The corresponding experimental data for $T^{*}$ in LSCO are also reported for comparison. We determine the coupling $V$ between the charge fluctuations and the quasiparticles, which is the only parameter for which an a priori estimate is difficult, by imposing that the fluctuation-corrected QCP is located at the $T=0$ extrapolation of the $T^{*}(\delta)$ curves. We used $\gamma=0.7 \mathrm{eV}^{-1}$ and $\gamma=0.4 \mathrm{eV}^{-1}$ for LSCO and Bi2212 respectively, and $V=0.54 \mathrm{eV}$ for both. It is worth noticing that, similarly to the case of $T_{C O}^{(0)}(\delta)$, the agreement between the calculated $T_{C O}(\delta)$ and the experimental points $T^{*}(\delta)$ is obtained without further adjustable parameters. We also notice that, contrary to the shift of $\delta_{c}$ at $T=0$, the slope of the curve $T_{C O}(\delta)$ is weakly dependent on $\omega_{0}$.
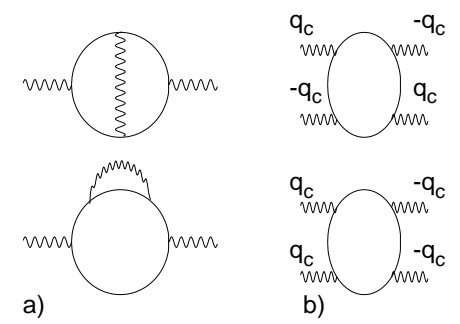

FIG. 2. (a) The two (vertex and selfenergy) corrections to the fermionic bubbles (solid line) due to critical charge fluctuations (wavy line). (b) Effective 4-leg vertices for the critical charge fluctuation field (wavy line) resulting from the integration over the fermion loops (solid line).

- Novel isotopic effects - The m-f weak-pseudogap crossover temperature $T_{C O}^{(0)} \sim T^{0}$ is determined by $\lambda$ only. Therefore, it does not depend on $\omega_{0}$ and is not expected to display any isotopic dependence. On the other hand, quantities determined by the fluctuations crucially involve $\omega_{0}$. New physical effects can then arise in the isotopic substitutions. Since $\omega_{0}$ decreases by increasing the ionic mass, the corrections to $\delta_{c}^{(0)}$ and to $T_{C O}^{(0)}(\delta)$ become smaller and the fluctuation-corrected quantities $\delta_{c}$ and $T_{C O}$ shift to higher doping, closer to the $\mathrm{m}-\mathrm{f}$ values, as shown in Fig. 3, where we report the line $T_{C O}(\delta)$, calculated via Eq. (价, with parameters to fit the $T^{*}$ data of LSCO (see above), together with its isotopic shift calculated for ${ }^{16} \mathrm{O} \rightarrow{ }^{18} \mathrm{O}$ substitution (i.e., for a five percent reduction of $\left.\omega_{0}\right)$. Correspondingly we expect that the portion of the curve $T_{c}(\delta)$ on the left of $T^{0}(\delta)$ is rigidly translated along the horizontal axis. The rational behind this translation is that the whole physics of these materials is essentially determined by the proximity to the critical line $T_{C O}(\delta)$ and to the related QCP. On the other hand, on the right of the m-f critical line $T_{C O}^{(0)}(\delta) \sim T^{0}(\delta)$ the fluctuations are small and the physical processes at $T>T^{0}(\delta)$ are captured by the m-f description, where 
$\lambda$ (not $\left.\omega_{0}\right)$ is relevant. As a consequence $T^{0}(\delta)$ and the portion of $T_{c}(\delta)$ near and above $T^{0}(\delta)$ are not expected to be shifted by IE's.

In the underdoped region, there are two evident consequences of the isotopic shift, which becomes more substantial: The shift upon reducing $\omega_{0}$ is negative (as usual) in $T_{c}$, but, contrary to standard theories based on CO pseudogap [21], it is positive in $T_{C O} \sim T^{*}$. Moreover, when the slope of $T_{C O}(\delta)$ is large, a rather small isotopic shift in $\delta_{c}$ can result in a substantial shift in $T_{C O} \sim T^{*}$. The steeper is $T^{*}$, the larger is the IE. On the other hand, since the curve $T_{c}(\delta)$ is rather flat, particularly in the optimal and moderately under-doped regimes, the expected IE on $T_{c}$ in these compounds is small in agreement with long-standing experiments [7,8]. This large difference in the IE for $T_{c}$ and $T^{*},\left(\Delta T_{c} / \Delta M\right) /\left(\Delta T^{*} / \Delta M\right) \ll 1$, is indeed experimentally observed in $\mathrm{HoBa}_{2} \mathrm{Cu}_{4} \mathrm{O}_{8}$ (HBCO124), and reported in Ref. [11], where it is also noticed that there is a "striking similarity between isotopic substitution and underdoping with respect to both $T_{c}$ and $T^{* \prime}$. Although we are not aware of any systematic analysis of the doping dependencies of $T_{c}, T^{*}$ and their isotopic shifts in HBCO-124, this observation finds its natural interpretation within our QCP scenario, where the isotopic substitution produces a shift of the QCP and is therefore nearly equivalent to underdoping.

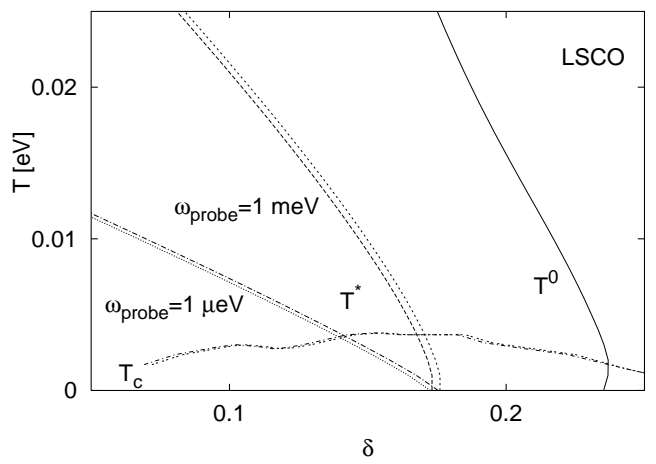

FIG. 3. Calculated effect of the isotopic change ${ }^{16} \mathrm{O} \rightarrow{ }^{18} \mathrm{O}$ (i.e. $\omega_{0}=70 \mathrm{meV}$ and $\omega_{0}^{\prime}=66 \mathrm{meV}$ ) on $T^{*}$ in LSCO, both for a fast- and a slow-probe measurement. The inferred shift on $T_{c}$ is also reported

The slope of $T_{C O}(\delta)$ increases by increasing $\omega_{\text {probe }}$, while the QCP is unshifted. Therefore, the IE on $T_{C O} \sim$ $T^{*}$ is enhanced, and we have the general trend that faster probes should detect a larger IE on $T^{*}$, since the effect of fluctuation diminishes. Although we cannot account for the near-absent or negative IE on $T^{*}$ within the almost static probes in $\mathrm{YBa}_{2} \mathrm{Cu}_{4} \mathrm{O}_{8}$,9, 10], this general trend is in qualitative agreement with a much stronger effect observed in the isostructural HBCO-124 with fast neutron scattering [11]. Indeed, this fast-probe experiment should be represented by the curve $T_{C O}(\delta)$ correspond- ing to $\omega_{\text {probe }}=1 \mathrm{meV}$ in Fig. 3. The huge IE on $T^{*}$ observed in HBCO-124 suggests that the curve $T_{C O}(\delta)$ in this system is steeper than in LSCO.

Upon underdoping, while $T_{c}$ decreases, its IE increses in agreement with a general experimental trend [7]. However, in the strongly underdoped materials, where a substantial IE in the penetration depth 23] and in the x-ray absorption [22] have been observed, our results can provide only qualitative indications, as other effects (magnetic, polaronic, lattice-pinning) not included in (II) become relevant. For this doping regime, a different (additional) interpretation of the IE in terms of a superconductor-to-insulator QCP has been recently proposed [24].

Acknowledgments. We acknowledge stimulating discussions with C. Castellani and K. A. Müller.

[1] For a summary of this evidences see, e.g., the Introduction of C. Castellani, C. Di Castro, and M. Grilli, Z. für Physik 103, 137 (1997); J. L. Tallon, et al., Phys. Stat. Sol. (b) 215, 531 (1999); J. L. Tallon, and J. W. Loram, Physica C 349, 53 (2001).

[2] M. Gutmann, E. S. Božin, and S. J. L. Billinge, condmat/0009141.

[3] A. J. Millis, H. Monien, and D. Pines, Phys. Rev. B 42, 167 (1990); P. Monthoux, A. V. Balatsky, and D. Pines, Phys. Rev. B 46, 14803 (1992); S. Sachdev and J. Ye, Phys. Rev. Lett. 69, 2411 (1992).

[4] C. M. Varma, Phys. Rev. Lett. 83, 3538 (1999).

[5] C. Castellani, C. Di Castro, and M. Grilli, Phys. Rev. Lett. 75, 4650 (1995).

[6] M. Vojta, Y. Zhang, and S. Sachdev, Phys. Rev. Lett. 85, 4940 (2000).

[7] J. P. Franck, in Physical Properties of High Temperature Superconductors $I V$, edited by D. M. Ginsberg (World Scientific, Singapore, 1994), p. 189.

[8] M. K. Crawford, et al., Phys. Rev. B 41, 282 (1990).

[9] F. Raffa, et al., Phys. Rev. Lett. 81, 5912 (1998).

[10] G. V. M. Williams, et al., Phys. Rev. B 61, R9257 (2000).

[11] D. Rubio Temprano, et al., Phys. Rev. Lett. 84, 1990 (2000).

[12] A. Perali, et al., Phys. Rev. B 54, 16216 (1996).

[13] F. Becca, et al., Phys. Rev. B 54, 12443 (1996).

[14] In our framework the CO instability is a second-order transition leading to a gradual increase of the charge modulation. It is only when one enters deeply inside the (locally) ordered phase that anharmonic distortions of the charge profile may arise from the enhanced interactions with the spin and the lattice degrees of freedom. This may lead to the stripe formation, as revealed in neutron scattering, see J. M. Tranquada, et al., Nature 375, 561 (1995).

[15] This feature is the natural consequence of the damping of the charge fluctuations due to the creation of particlehole pairs at arbitrarily low energy when $\mathbf{q}_{c}$ joins two "hot" points on the Fermi surface.

[16] G. Seibold, et al., Eur. Phys. J. B 13, 87 (2000).

[17] H. Ding, et al., J. Phys. Chem. Solids 59, 1888 (1998).

[18] K. Ishida, et al., Phys. Rev. B 58, R5960 (1998).

[19] J. A. Hertz, Phys. Rev. B 14, 1165 (1976). 
[20] A. J. Millis, Phys. Rev. B 48, 7183 (1993).

[21] I. Eremin, et al., Phys. Rev. B 56, 11305 (1997).

[22] A. Lanzara, et al., J. Phys.: Condens. Matter 11, L541
(1999).

[23] J. Hofer, et al., Phys. Rev. Lett. 84, 4192 (2000).

[24] T. Schneider and H. Keller, cond-mat/0011381. 